NOTA PALEONTOLÓGICA

\title{
Coprolitos en la Formación Monte Hermoso (Mioceno tardío-Plioceno temprano), Farola Monte Hermoso, Buenos Aires, Argentina
}

\author{
Rodrigo L. TOMASSINI1,2 y Claudia I. MONTALVO²
}

\section{Introducción}

Los bromatolitos forman parte de un grupo de trazas fósiles que incluye a los coprolitos o excrementos fósiles, las egagrópilas o regurgitaciones y los cololitos, es decir restos que contienen material retenido "in situ" dentro del tracto intestinal (Hunt et al., 1994). Al igual que otras trazas constituyen una evidencia directa del comportamiento de antiguos organismos, aportando información referida a paleodietas, diversidad de biotas y relaciones interespecíficas tales como predador-presa y parasitosis, entre otras (Pollard, 1990; Andrews y Fernández Jalvo, 1998; Chin, 2002; Duarte et al., 1999).

Los coprolitos producidos por vertebrados son notablemente variables en composición, forma y tamaño, dependiendo de la masa corporal del productor, tipo de alimentación y condiciones de depositación, diagénesis y preservación a las que fueron sometidos. Estas características dificultan la asignación de los mismos a un productor en particular (Häntzschel et al., 1968; Pollard, 1990; Chin, 2002).

En esta oportunidad se describen y analizan tres coprolitos, con material fósil muy fragmentario incluido en ellos, provenientes de los niveles inferiores de la Formación Monte Hermoso (Zavala, 1993). Sobre la base de sus características se definieron dos morfotipos distintos.

\section{Ubicación geográfica y marco geológico}

Farola Monte Hermoso está ubicada al sudeste de la provincia de Buenos Aires, en cercanías del balneario Pehuen Co y a unos $53 \mathrm{~km}$ al sur-sudeste de la ciudad de Bahía Blanca (figura 1.1). Constituye la localidad tipo de la Formación Monte Hermoso

1CONICET.rodher23@yahoo.com.ar

2Facultad de Ciencias Exactas y Naturales, UNLPam. Avenida Uruguay 151, 6300 Santa Rosa, La Pampa, Argentina.

cmontalvo@exactas.unlpam.edu.ar

๑)Asociación Paleontológica Argentina
(Zavala, 1993) y del Piso/Edad Montehermosense y Chapadmalalense inferior (Cione y Tonni, 2005).

Los tres ejemplares analizados provienen del sector inferior de la barranca, a unos $30 \mathrm{~cm}$ de la base, en niveles de areniscas finas y areniscas limosas color castaño claro a rojizo de la Formación Monte Hermoso (figura 1.2). Desde el punto de vista litoestratigráfico, los niveles portadores son asignables a la facies Fl de Zavala y Navarro (1993), correspondiente a depósitos de llanura de inundación y correlacionables con la Biozona de Trigodon gaudryi Ameghino, 1882, base bioestratigráfica del Piso/Edad Montehermosense (Mioceno tardío-Plioceno temprano; Cione y Tonni, 2005).

\section{Materiales y métodos}

Los materiales están depositados en el Museo Municipal de Ciencias Naturales "Carlos Darwin" de Punta Alta, bajo las siglas MD-FM-08-36, MD-FM08-37 y MD-FM-08-38 y fueron medidos con calibre mecánico de precisión de $0,05 \mathrm{~mm}$. Los tres coprolitos se identificaron y describieron siguiendo los criterios propuestos por distintos autores para definir este tipo de trazas (Häntzschel et al., 1968; Andrews, 1990; Hunt et al., 1994; Chame, 2003). Se fotografiaron y se describieron sus características. Posteriormente se extrajeron los restos óseos en los casos posibles, se evaluaron los elementos esqueléticos (NR: número de restos) y fragmentos indeterminables. Se realizó la determinación anatómica y taxonómica y se describieron sus características tafonómicas, utilizando la metodología propuesta por Andrews (1990) y Fernández Jalvo y Andrews (1992), utilizando un microscopio binocular Leica Ms5. Los ejemplares más significativos por sus características y el material aglutinante (matriz) fueron fotografiados con microscopio electrónico de barrido (SEM) JEOL 35 CF. La estructura interna de los coprolitos y su composición mineralógica se determinó mediante secciones delgadas y la composición elemental a partir de análisis de EDX (Energy Dispersive $X$-ray), utilizando un EDAX modelo DX-4.

AMGHB2-0002-7014/10\$00.00+.50 


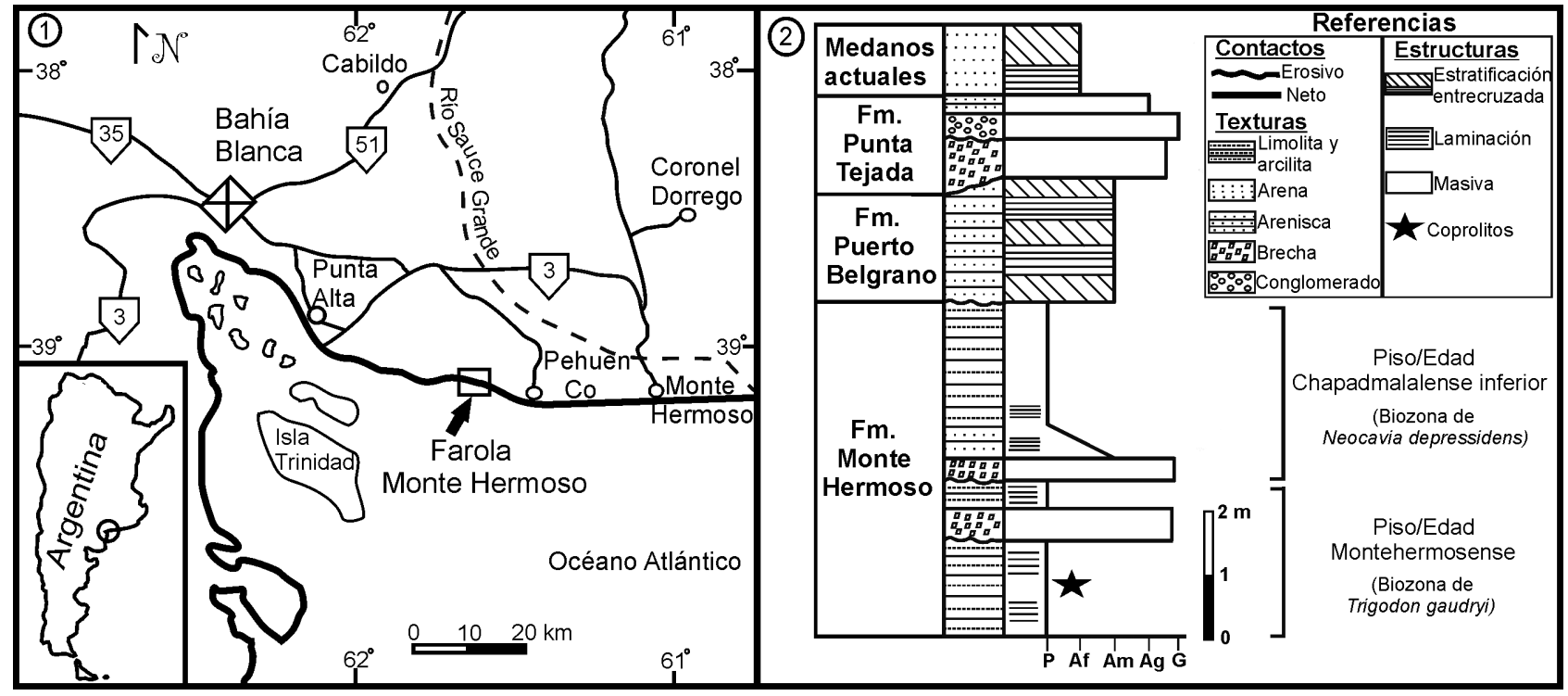

Figura 1. 1, Mapa de ubicación geográfica de Farola Monte Hermoso / location map of Farola Monte Hermoso. 2, Perfil estratigráfico de Farola Monte Hermoso. Modificado de Zavala (1993) / Stratigraphic section of Farola Monte Hermoso. Modified from Zavala (1993).

\section{Resultados}

De acuerdo a sus características, los tres coprolitos fueron divididos en dos morfotipos diferentes, denominados I y II.

Los dos especímenes del morfotipo I (MD-FM-0836 y MD-FM-08-37) fueron identificados rápidamente en el campo por tratarse de pequeñas acumulaciones de fósiles muy fragmentados, con elevada densidad y de coloración muy oscura debido a la fuerte impregnación por óxidos, que en planta presentaban diferencias de color y grado de cementación respecto de la matriz que las contenía, de tal modo que definían una superficie ovalada.

El ejemplar MD-FM-08-36 (figura 2.1) estaba dividido en 4 sectores subelípticos de diferentes tamaños, con diámetros máximos variables entre 10 y $50 \mathrm{~mm}$ y espesor de $5 \mathrm{~mm}$ como máximo. Se recuperaron 284 fósiles muy rotos, de los cuales el 91,20\% eran indeterminables. El tamaño de éstos no superó los $9 \mathrm{~mm}$ de largo y $3 \mathrm{~mm}$ de ancho. El 8,80\% restante consistió en elementos craneanos y postcraneanos con distintos grados de rotura. Si bien no se observó un predominio marcado de ningún elemento, los más abundantes fueron las vértebras $(\mathrm{NR}=8)$ y las diáfisis de huesos largos indeterminados ( $\mathrm{NR}=7$ ).

Se identificaron restos asignados a Paedotherium sp. (Notoungulata, Hegetotheriidae), Rodentia indet., Mammalia indet. y Osteichthyes indet.

Los restos presentaban evidencias de redondeamiento en las zonas de fractura y áreas salientes, grados de rotura altos y fracturas espiraladas (Marshall, 1989) (figura 3.1). Utilizando la metodología pro- puesta por Fernández Jalvo y Andrews (1992), la evaluación de los grados de corrosión por digestión fue moderada en un fémur proximal (Mammalia indet.), leve en un fragmento de incisivo (Rodentia indet.) y ausente en un premolar (Paedotherium sp.).

La muestra MD-FM-08-37 (figura 2.2) era subelíptica, con un diámetro máximo de $77 \mathrm{~mm}$ y mínimo promedio de $35 \mathrm{~mm}$. El espesor de la acumulación no superó los $5 \mathrm{~mm}$. Se recuperaron 134 fósiles, todos muy fragmentados, de los cuales el 79,85\% resultaron indeterminables, con tamaños máximos de $6 \mathrm{~mm}$ de largo y 3,5 mm de ancho. El 20,15\% restante correspondió principalmente a molares aislados, $(\mathrm{NR}=4)$ y fragmentos de diáfisis $(\mathrm{NR}=9)$ y epífisis $(\mathrm{NR}=7)$ de huesos largos indeterminados.

Se identificaron restos de Mammalia indet., Rodentia indet. y molares de Actenomys priscus Owen, 1840 (Rodentia, Octodontidae) con características que indican la presencia de, al menos, un individuo adulto y un juvenil.

Todos los fósiles mostraron evidencias de redondeamiento y un grado de rotura extremo, con predomino de fracturas espiraladas. Además fue común la presencia de sectores de la superficie con cambios de color, manifestados como áreas blancas y pardo oscuras.

Se observaron evidencias leves de corrosión por digestión en la cara oclusal del molar de un individuo juvenil de Actenomys priscus; digestión leve a moderada en siete epífisis de huesos largos indeterminados de Mammalia indet. y digestión fuerte en un fragmento de fémur proximal de un Mammalia indet. (figura 3.2). 


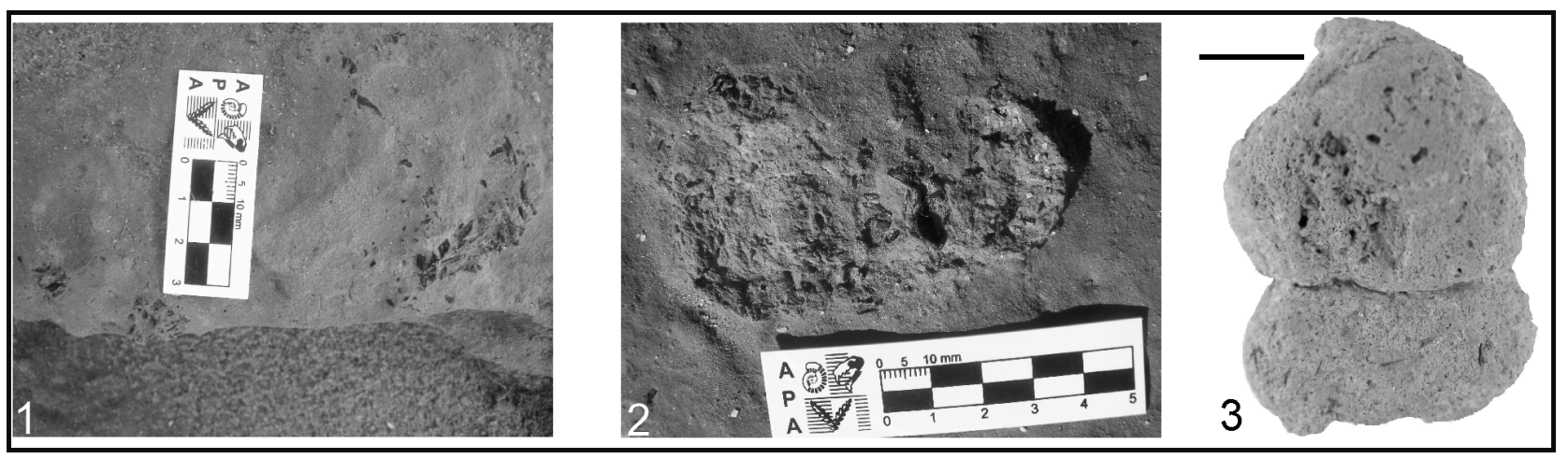

Figura 2. 1, Coprolito MD-FM-08-36, morfotipo I / Coprolite MD-FM-08-36 morphotype I. 2, Coprolito MD-FM-08-37, morfotipo I / Coprolite MD-FM-08-37 morphotype I. 3, Coprolito MD-FM-08-38, correspondiente al morfotipo II / Coprolite MD-FM-08-38, morphotype II. Escala / Scale bar $=0,5 \mathrm{~cm}$.

El EDX realizado sobre la matriz y los fósiles evidenció la presencia de fósforo y calcio como elementos predominantes, además de sílice, aluminio, magnesio, sodio y cloro, en menor proporción. Por otro lado, se determinó que la coloración oscura que poseen estos materiales se debe a impregnaciones de óxidos de manganeso.

El ejemplar del morfotipo II (MD-FM-08-38) (figura 2.3) presenta la matriz cementada, es de color pardo amarillento, con un extremo plano y el otro finalizando en punta. La longitud es de $21 \mathrm{~mm}$ y el diámetro promedio de $14,5 \mathrm{~mm}$, con sección transversal subcircular. En la zona media presenta una sutura que divide al resto en dos segmentos de tamaños subiguales. Las secciones delgadas evidenciaron la presencia de una matriz de grano muy fino, en la cual se distribuyen pequeños cristales de cuarzo, mafitos, plagioclasas y una importante cantidad de astillas óseas. El tamaño máximo de estas últimas es de 5 $\mathrm{mm}$ de largo y $2 \mathrm{~mm}$ de ancho; algunas presentan sus bordes redondeados y coloración oscura por impregnación de óxidos. En algunos casos, se observó la preservación de parte de la estructura original del hueso.

Si bien no se observaron estriaciones en la superficie externa del coprolito, se destaca la presencia de abundantes cavidades subcirculares.

Los diagramas de EDX evidencian que el fósforo y el calcio son los elementos dominantes en la muestra. En algunos casos, y en menor proporción que los anteriores, también se determinó la presencia de sílice, aluminio, hierro y magnesio.

\section{Discusión y conclusiones}

La mayoría de las características tafonómicas que presentaron los restos de las dos acumulaciones del coprolito morfotipo I (MD-FM-08-36 y MD-FM-08-
37) difieren notablemente de las de los fósiles hallados en la facies de llanura de inundación de la Formación Monte Hermoso. Como se indicó, se trata de áreas reducidas con una densidad muy alta de fragmentos fósiles, la mayoría indeterminables. Sin embargo, para el tamaño reducido de cada acumulación, la identificación de algunos restos mostró una diversidad taxonómica elevada. Todos los materiales incluidos presentaban un alto grado de rotura y redondeamiento de bordes y superficies de fractura, y varios de ellos tenían evidencias de corrosión por acción digestiva. Estas características permitieron descartar la posibilidad de que algún agente físico estuviera asociado al origen de las mismas.

Los distintos atributos tafonómicos analizados, sumados a las características de la matriz de cada acumulación, diferente respecto de la roca hospedante, avalaron su asignación a acumulaciones generadas por un carnívoro. Por las características tafonómicas mencionadas, ambas acumulaciones podrían atribuirse a un carnívoro que produce modificaciones de moderadas a fuertes en los huesos de sus presas. Las heces de mamíferos carnívoros y las egagrópilas de rapaces diurnas se caracterizan por incluir restos óseos fragmentados y digeridos, con bordes redondeados y ocasionalmente con marcas en su superficie (Andrews, 1990; Mondini, 2000; Gómez, 2005, 2007; Montalvo et al., 2007, 2008; Montalvo y Tallade, 2009).

Sin embargo, la escasez de elementos anatómicos determinables en cada una de las acumulaciones, impide realizar un análisis exhaustivo de la representación anatómica que permita comparar los datos aquí obtenidos con otros de acumulaciones en egagrópilas o heces actuales producidas por diferentes carnívoros. En ese sentido, clásicamente las heces de mamíferos carnívoros constituyen el tipo de acumulación con mayor cantidad de restos indeterminables ya que, previo a la acción digestiva destruyen parte de

AMEGHINIANA 47 (1), 2010 


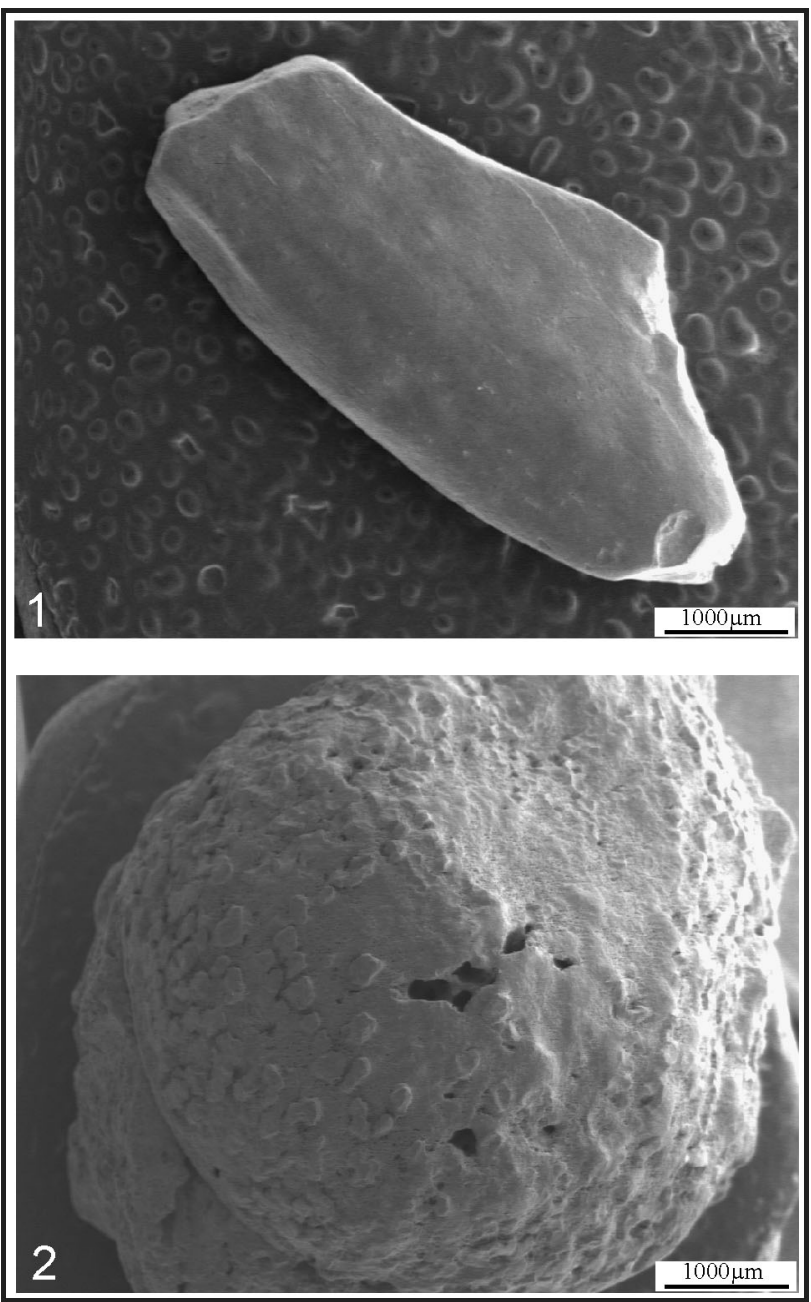

Figura 3. 1, Fragmento de hueso indeterminado con evidencias de redondeamiento en los bordes / fragment of indeterminate bone with evidences of rounding on the edges. 2, Fémur proximal de Mammalia indet. con evidencias de corrosión digestiva fuerte / Mammalia indet. proximal femur with evidences of high digestive corrosion.

los elementos esqueléticos durante la masticación (Mellet, 1974; Korth 1979; Andrews, 1990).

La distribución matriz-soportada que presentan los fragmentos fósiles avala la asignación de estas dos acumulaciones a coprolitos, tal como lo exponen Chin et al. (1998) y permite rechazar la idea de que se trate de egagrópilas fósiles.

Un atributo diagnóstico que define a los coprolitos de carnívoros, además de la presencia de gran cantidad de fragmentos de huesos, es su composición fosfática, con elevados porcentajes en el contenido de fósforo y calcio (Bradley, 1946; Chin et al., 1998; Hunt et al., 1994). Los análisis de EDX determinaron la composición fosfática en las dos acumulaciones, con el fósforo y calcio como elementos principales. La ocurrencia de fósforo y calcio en la matriz es coherente con la composición fosfática cálcica obtenida también en los fósiles. Esta característica también permitiría asociar a ambas acumulaciones con coprolitos y diferenciarlas de las egagrópilas las cuales, si bien también presentan una gran abundancia de fragmentos óseos, se caracterizan por la ausencia de una matrix fosfática (Pollard, 1990).

Las impregnaciones por manganeso que presentan los restos constituyen un carácter que los asemeja a otros hallados en el mismo nivel. Lo mismo ocurre con los cambios de color que afectan pequeñas superficies de algunos de los restos de las acumulaciones y que son interpretados como producto de acción corrosiva diagenética.

Finalmente, el poco espesor de las acumulaciones asignadas al morfotipo I (MD-FM-08-36 y MD-FM08-37) sugeriría que se trata de coprolitos colapsados. El colapso podría haber ocurrido por pisoteo o por carga litostática.

En el caso del ejemplar correspondiente al morfotipo II (MD-FM-08-38), sus características morfométricas, texturales y la presencia de fragmentos fósiles indeterminados permiten interpretarlo como un coprolito producido por un mamífero carnívoro (Häntzschel et al., 1968; Chin, 2002; Chame, 2003). Avalan esta asignación otros caracteres, como un extremo aguzado interpretado como una marca de extrusión, una línea de sutura en la zona media que define la unión de las dos masas que forman el coprolito y cavidades subredondeadas, atribuibles a estructuras de escape de burbujas gaseosas. No se identificaron estriaciones relacionables a marcas de pelo. Esta asignación también está confirmada por los resultados de los análisis de EDX, los cuales determinaron la composición fosfática del material, con el fósforo y calcio como elementos dominantes.

La composición fosfática, seguramente derivada de la disolución de la apatita de los huesos consumidos (Bradley, 1946; Häntzschel et al., 1968; Hunt et al., 1994), sumado al tipo de ambiente sedimentario en el cual fueron depositados (llanura de inundación) favoreció la preservación de los tres ejemplares y sugiere que los mismos no han sufrido ningún tipo de transporte (Verde y Ubilla, 2002).

La presencia de Actenomys priscus y Paedotherium sp., entre los restos incluidos en las dos acumulaciones del morfotipo I avalaría la coetaneidad de las mismas con la fauna del nivel portador, que clásicamente ha sido asignada al Montehermosense (Mioceno tardío-Plioceno temprano).

Teniendo en cuenta la sumatoria de características presentes, tanto en las dos acumulaciones correspondientes al morfotipo I como así también en el ejemplar del morfotipo II, los restos son asignados a coprolitos producidos por mamíferos carnívoros cuya identidad y tamaño estimado no es posible ajustar con la evidencia disponible.

Estos hallazgos constituyen la primera evidencia 
clara de la acción de mamíferos carnívoros (depredación o carroñeo) en niveles de la asociación faunística de la Formación Monte Hermoso y aportan información acerca de las distintas interacciones tróficas en este paleoecosistema.

\section{Agradecimientos}

A los revisores (M. Verde y F. Martin), cuyos comentarios y correcciones enriquecieron el trabajo y a R. Melchor por sus sugerencias. La Facultad de Ciencias Exactas y Naturales, Universidad Nacional de La Pampa, financió parte de este trabajo.

\section{Bibliografía}

Ameghino, F. 1882. Catálogo explicativo de las colecciones de antropología, prehistoria y de paleontología de Florentino Ameghino. Catálogo de la Sección de la provincia de Buenos Aires en la Exposición Continental Sudamericana. Anexo A: 35-42. Buenos Aires

Andrews, P. 1990. Owls, caves and fossils. Predation, preservation and accumulation of small mammal bones in caves, with the analysis of the Pleistocene cave faunas from Westburysub-Mendip, Somerset, UK. Natural History Museum Publication, London, $231 \mathrm{pp}$.

Andrews, P. y Fernández Jalvo, Y. 1998. 101 uses for fossilized faeces. Nature 39: 629-630.

Bradley, W.H. 1946. Coprolites from the Bridger Formation of Wyoming: their composition and microorganisms. American Journal of Science 244: 215-239.

Chame, M. 2003. Terrestrial mammal feces: a morphometric summary and description. Memórias do Instituto Oswaldo Cruz 98: 71-94.

Cione, A. y Tonni, E. 2005. Bioestratigrafía basada en mamíferos del Cenozoico superior de la provincia de Buenos Aires, Argentina. En: R.E. de Barrio, R.O. Etcheverry, M.F. Caballé y E. Llambías (eds.), Geología y Recursos Minerales de la Provincia de Buenos Aires. $16^{\circ}$ Congreso Geológico Argentino (La Plata), Relatorio, 11: 183-200.

Chin, K. 2002. Analyses of coprolites produced by carnivorous vertebrates. Paleontological Society papers 8: 43-49.

Chin, K., Tokaryk, T., Erickson, G. y Calk, L. 1998. A king-sized theropod coprolite. Nature 393: 680-682.

Duarte, A.N., Verde, M., Ubilla, M., Araújo, A., Martins, P.C., Reinhard, K., y Ferreira, L.F. 1999. Note on parasite eggs in mineralized Carnivora coprolites from the Upper Pleistocene Sopas Formation, Uruguay. Paleopathology Newsletter 107: 6-8.

Fernández Jalvo, Y. y Andrew, P. 1992. Small mammal taphonomy of Gran Dolina, Atapuerca (Burgos), Spain. Journal of Archaeological Science 19: 407-428.

Gómez, G.N. 2005. Analysis of bones modifications of Bubo virginianus pellets from Argentina. Journal of Taphonomy 3: 1-16.

Gómez, G.N. 2007. Predators categorizations based on taphonomic analysis of micromammals bones: a comparison to proposed models. En: M.A. Gutierrez, L. Miotti, G. Barrientos, G. Mengoni Goñalons y M. Salamme (eds). Taphonomy and Zooarqueology in Argentina. B AR, International Series, 1601, Oxford, UK, $233 \mathrm{pp}$.
Häntzschel, W., El-Baz, F. y Amstutz, G. 1968. Coprolites, an annotated bibliography. Memoirs of the Geological Society of America 108: 1-132.

Hunt, A.P., Chin, K. y Lockley, M.G. 1994. The Paleobiology of vertebrate coprolites. En: S.C. Donovan (ed.), The Paleobiology of trace fossils, John Wiley, London, pp. 221-240.

Korth, W. 1979. Taphonomy of microvertebrate fossil assemblages. Annals of Carnegie Museum 48: 235-285.

Marshall, L. 1989. Bone modification and "The laws of burial". En: R. Bonnichsen y M. Sorg (eds.), Bone modification, People of the Americas Publications. University of Main, 535 pp.

Mellet, J.S. 1974. Scatological origin of microvertebrate fossil accumulations. Science 185: 349-350.

Mondini, N.M. 2000. Tafonomía de abrigos rocosos de la Puna. Formación de conjuntos escatológicos por zorros y sus implicancias arqueológicas. Archaeofauna 9: 151-164.

Montalvo, C.I. y Tallade, P.O. 2009. Taphonomy of the accumulations produced by Caracara plancus (Falconidae). Analysis of prey remains and pellets. Journal of Taphonomy 7: 235-248.

Montalvo, C.I., Pessino, M. y González, V. 2007. Taphonomic analysis of remains of mammals eaten by pumas (Puma concolor Carnivora, Felidae) in central Argentina. Journal of Archaeological Science 34: 2151-2160.

Montalvo, C.I., Pessino, M.E. y Bagatto, F.C. 2008. Taphonomy of the bones of rodents consumed by Andean hog-nosed skunks (Conepatus chinga, Carnivora, Mephitidae) in central Argentina. Journal of Archaeological Science 35: 1481-1488.

Pollard, J.E. 1990. Evidence for diet. En: D.E.G. Briggs y P.R. Crowther (eds.), Paleobiology. A Synthesis Synthesis, Blackwell, London, $500 \mathrm{pp}$.

Verde, M. y Ubilla, M. 2002. Mammalian carnivore coprolites from the Sopas Formation (Upper Pleistocene, Lujanian stage), Uruguay. Ichnos 9: 77-80.

Zavala, C. 1993. Estratigrafía de la localidad de Farola Monte Hermoso (Plioceno-reciente). Provincia de Buenos Aires. $12^{\circ}$ Congreso Geológico Argentino y $2^{\circ}$ Congreso de Exploración de Hidrocarburos (Mendoza), Actas 2: 228-235.

Zavala, C. y Navarro, E. 1993 Depósitos fluviales en la Formación Monte Hermoso (Plioceno inferior-medio). Provincia de Buenos Aires. $12^{\circ}$ Congreso Geológico Argentino y $2^{\circ}$ Congreso de Exploración de Hidrocarburos (Mendoza), Actas 2: 236-244.

Recibido: 16 de abril de 2009 .

Aceptado: 31 de agosto de 2009. 